\title{
FLORISTICS AND PHYTOSOCIOLOGY OF THE GALLERY FOREST OF THE BACABA STREAM, NOVA XAVANTINA, MATO GROSSO, BRAZIL
}

\author{
B. S. Marimon*, J. M. Felfili† \& E. S. Lima*
}

\begin{abstract}
The study was carried out on the gallery forest of the Bacaba stream situated in the Municipal Ecological Reserve 'Mário Viana' ( $\left.14^{\circ} 43^{\prime} \mathrm{S}, 52^{\circ} 21^{\prime} \mathrm{W}\right)$ in Nova Xavantina, Eastern Mato Grosso, Brazil. Three sections of the gallery (upper, middle and lower) running downstream and differing in slope were surveyed by stratified sampling. Fortyseven nested $10 \mathrm{~m} \times 10 \mathrm{~m}$ plots were analysed in each section, giving a total sampling area of 1.41 ha overall. All trees or lianas $\geq 15 \mathrm{~cm}$ girth at breast height were recorded and a total of 129 species belonging to 105 genera and 47 families were found.

Diversity was high, with the Shannon index ranging from 3.84 nats/individual in the lower section to 4.08 in the middle section. The most important families (IVI) were Caesalpiniaceae (upper and middle sections) and Arecaceae (lower section), and the most important species were Diospyros obovata (upper section), Hymenaea courbaril var. stilbocarpa (middle section) and Mauritia flexuosa (lower section). Morisita and Sørensen indices of similarity were calculated. The floristic composition was complex and included species in common with a number of Brazilian forest types and with cerrado (savanna), as well as many widespread species, but stronger links with Amazonian forests could be detected. This is to be expected since the area lies in the ecotonal zone of the cerrado and Amazonian forest biomes and the Bacaba stream itself is a tributary of the Mortes-Araguaia-Amazon river system.
\end{abstract}

Keywords. Amazon, cerrado, gallery forests, phytosociology, savanna.

\section{INTRODUCTION}

Gallery forests in the cerrado biome of Central Brazil are generally evergreen mesophytic formations occurring alongside watercourses, and are surrounded by savanna vegetation (cerrado or campo). In spite of forming a dendritic network throughout the region, gallery forests cover only $5 \%$ of the 2 million $\mathrm{km}^{2}$ of the cerrado biome. They show high species diversity (Felfili, 1995) and have floristic links with the Amazonian and Atlantic rainforests (Oliveira-Filho \& Ratter, 1995). Their flora also contains some elements of the cerrado (Oliveira-Filho et al., 1994a,b) and a few endemic species. Environmental heterogeneity within the forest is high (Brinson, 1990) and mosaic patterns occur where communities related to the stages of a soil

\footnotetext{
* Universidade do Estado de Mato Grosso, Campus Universitário de Nova Xavantina, Departamento de Ciências Biológicas, Caixa Postal 08, 78690-000 Nova Xavantina-MT, Brazil.

$\dagger$ Universidade de Brasília, Departamento de Engenharia Florestal, 70919-970 Brasília-DF, Brazil.

This project received financial support from the Foundation to Support Research in Mato Grosso State (FAPEMAT).
} 
humidity gradient can be identified (Felfili, 1995; Felfili et al., 1998; Silva-Júnior et al., 1998). The presence of gaps, the edge effect caused by the sharp boundaries with cerrado and campo vegetation, and occasional fires play an important role in the maintenance of the high diversity (Felfili, 1995, 1997; Kellman \& Meave, 1997).

Gallery forests are of vital importance in hydrographic basins in controlling water flow, and retaining sediments and chemical nutrients. They also provide food and habitat for much of the fauna of the cerrado biome and provide corridors for the migration of animals. Their value is recognized in Brazilian laws prohibiting their destruction; however, the present advancement of agricultural and urban frontiers, together with weak enforcement of the current legislation, is threatening their existence.

A few studies have already been conducted in the gallery forests of Eastern Mato Grosso (Ratter et al., 1973, 1978; Felfili et al., 1998) and have shown their high diversity. The objective of this study is to provide further data by analysing the floristic composition and structure of the Bacaba gallery forest.

\section{LOCATION AND Methods}

\section{Study site}

The study was carried out in the Bacaba gallery forest in the Municipal Ecological Reserve 'Mário Viana' $\left(14^{\circ} 43^{\prime} \mathrm{S}, 52^{\circ} 21^{\prime} \mathrm{W}\right)$ in Nova Xavantina, Eastern Mato Grosso, Brazil (Fig. 1).

The Mário Viana Reserve covers approximately 500ha at an average altitude of $250 \mathrm{~m}$, and contains several vegetation types characteristic of the cerrado biome (Brazilian savanna). The main vegetation type in the Reserve is cerrado sensu stricto (savanna woodland) (Marimon et al., 1998) but there are areas covered with campo (grasslands) and cerradão (dense savanna woodland), and a gallery forest occurs along the Bacaba stream at the west edge of the Reserve. The climate belongs to Köppen's subtype Aw, with 6-8 months of rain (annual precipitation 1300-1500mm) and a mean monthly temperature of $25^{\circ} \mathrm{C}$ (Camargo, 1963; Cochrane et al., 1985) (Fig. 2). The present study was restricted to the gallery forest where the predominant soils are lithosols and alluvium.

Three sections of the forest running downstream and differing in slope were chosen for this study. The upper section is characterized by the presence of quartzite rocks and lithosols and has a $10 \mathrm{~m}$ waterfall that flows rapidly during the rainy season. Here the forest lies in a valley with an average slope of $42 \%$. The middle section is also rocky and the soils are lithosols but the topography is less steep, with an average slope of $32 \%$. Rocks are absent in the lower section, where the soil is alluvium and the average slope is $5 \%$. The distance between the upper and middle sections was $150 \mathrm{~m}$ and between the middle and lower $200 \mathrm{~m}$.

The environmental heterogeneity of the forest is high due especially to variations in drainage related to the steep relief and abundance of rocky outcrops in the middle 


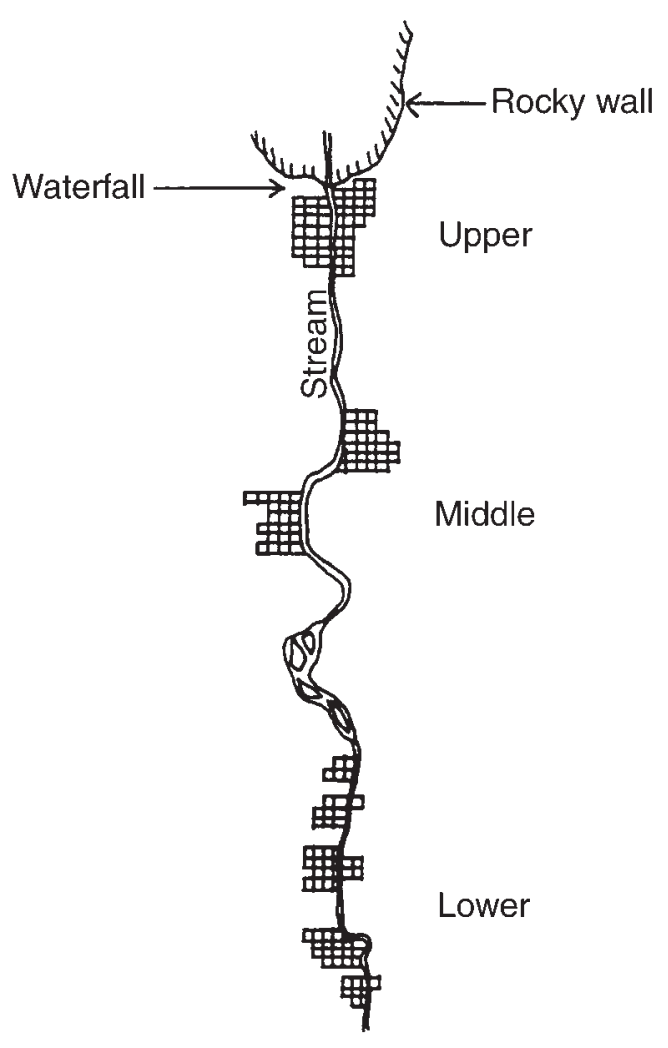

FIG. 1. Location of the plots in the three sections (upper, middle and lower) of the Bacaba gallery forest.

and upper sections and to the flat relief without rocks in the lower section. A large volume of water flows in the upper section during the rainy season and overflows the stream bed during heavy rains, but the drainage is generally rapid. In the steeper sites of the middle section the conditions are similar to the upper, but on more shallowly sloping areas water floods over the surface during the rainy season. In the lower section, the water-table lies close to the surface throughout the year and there is often flooding in the rainy season.

The vegetation surrounding the gallery forest varies from cerrado sensu stricto on the steeper sites with well-drained soil to campo limpo (grassland) where the terrain is flat and the soil badly drained throughout the year.

\section{Vegetation inventory}

Sampling was stratified as described by Philip (1994). In each section of the gallery forest (upper, middle and lower), 47 contiguous permanent plots $100 \mathrm{~m}^{2}(10 \mathrm{~m} \times 10 \mathrm{~m})$ 


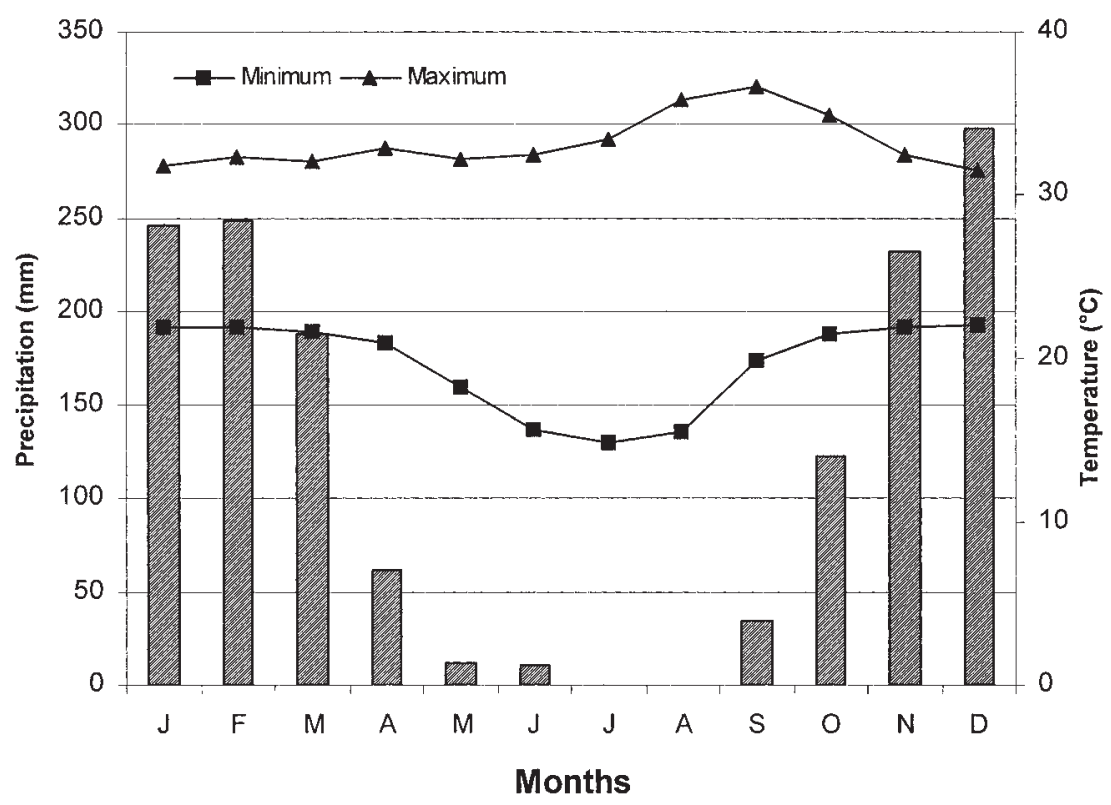

FIG. 2. Mean monthly precipitation and minimum and maximum temperature (1995-99) in the Bacaba gallery forest. Observations of the Climatological Station, Campus Universitário de Nova Xavantina, Nova Xavantina, Mato Grosso, Brazil.

were established, giving a total sampling area of 1.41 ha. The plots were arranged in lines at right angles to the stream, running from the stream bank to the forest edge (Fig. 1).

All trees and lianas with girth at breast height $(\mathrm{gbh}) \geq 15 \mathrm{~cm}$ were labelled with permanent aluminium tags, identified, and measured. Girth was measured to the nearest $1 \mathrm{~mm}$ with a tape, and a pole was used to measure height of the trees and lianas. Dead standing trees were also recorded. Voucher specimens were collected for identification and incorporated in the Herbarium NX, James Alexander Ratter Collection at the Nova Xavantina campus of the University of Mato Grosso State (UNEMAT).

\section{Data analysis}

The usual phytosociological parameters based on density, dominance and frequency (Curtis \& McIntosh, 1950, 1951), Shannon's diversity index $\left(\mathrm{H}^{\prime}=-\Sigma(p i \ln p i)\right.$, where $p i=n i / N, n i$ is the number of individuals of species $i$ and $N$ is the total number of individuals) and Simpson's index $(\lambda=\Sigma n i(n i-1) / N(N-1))$, were used to evaluate species importance and diversity in each section and for the complete surveyed forest area. Pielou's evenness index $\left(\mathrm{J}^{\prime}=\mathrm{H}^{\prime} / \ln (S)\right.$, where $S$ is the number of species) was also calculated (Magurran, 1988).

Morisita's similarity index was calculated to compare each forest section. This 
index is based on Simpson's index and varies between 0 and 1, values higher than 0.5 suggesting high similarity between communities (Brower \& Zar, 1977). The three sections were also compared using Sørensen's coefficient of similarity:

$(2 \times$ No. of spp. in common)/(No. of spp. at locality A and No. at locality B).

\section{RESUlts}

There were 129 species belonging to 105 genera in 47 families in the total forest area surveyed. Table 1 gives the overall species list and scores the occurrence of species in each section. The richest families were Fabaceae (8 species), Caesalpiniaceae (8), Apocynaceae (7), Rubiaceae (7) and Mimosaceae (6). The diversity indices and evenness for the three sections and for the total sampled area are given in Table 2.

Species occurrence and the phytosociological parameters of density, dominance and frequency for each section are given in Tables 3-5. Burseraceae was the most important family and Caesalpiniaceae the second in the upper section, representing $10.15 \%$ and $9.70 \%$ of total IVI respectively. The order of these two families was reversed in the middle section, with Caesalpiniaceae at 15.4\% and Burseraceae at $9.60 \%$. Arecaceae had the highest IVI in the lower section, representing $23.6 \%$ of the total. Most families were common to the three sections (Table 1).

\section{Upper section}

The absolute density was 1023 individuals/ha and basal area was $20.44 \mathrm{~m}^{2} / \mathrm{ha}$. Dead individuals represented $5 \%$ of the total density and $4.09 \%$ of the basal area. The most important species (Table 3) were Diospyros obovata (IVI=22.9), Calophyllum brasiliense (12.4), Tetragastris altissima (12.4), Protium heptaphyllum (11.7) and Astrocaryum vulgare (10.9), which together represented $23.5 \%$ of the total IVI. For D. obovata, relative density ( $\mathrm{RD}=9.8 \%$ ) was the most important component of IVI composition, and for $C$. brasiliense the most important was relative dominance $(\mathrm{RDo}=7.5 \%)$. Fifteen species were represented by single individuals. Diospyros obovata (with a relative frequency ( $\mathrm{RF}$ ) of $5.9 \%$ ) and T. altissima $(4.8 \%)$ were the most constant species in the sampling. Only nine species occurred in $20 \%$ or more plots, while 17 occurred only in single plots.

Amongst the most important species, Calophyllum brasiliense, Apuleia leiocarpa and Hymenaea courbaril var. stilbocarpa are emergents, with some trees reaching $25 \mathrm{~m}$. On the other hand, all trees of Astrocaryum vulgare are under $10 \mathrm{~m}$, while understorey species such as Siparuna guianensis and Tococa formicaria reach only $5 \mathrm{~m}$.

\section{Middle section}

The absolute density was 962 individuals/ha and basal area $22.28 \mathrm{~m}^{2} /$ ha. Dead individuals represented $2.43 \%$ of the total density and $2.84 \%$ of the basal area. The most important species in IVI (Table 4) were Hymenaea courbaril var. stilbocarpa (15.3), Tetragastris altissima (12.9), Apuleia leiocarpa (12.3), Pouteria torta (11.8) and 
TABLE 1. List of species occurring in the three forest sections studied in the Bacaba gallery forest, Nova Xavantina, Mato Grosso, Brazil

\begin{tabular}{|c|c|c|c|c|}
\hline Species & Family & Upper & Middle & Lower \\
\hline 1. Abuta grandifolia (Mart.) Sandw. & Menispermaceae & & & $\mathrm{x}$ \\
\hline 2. Acosmium sp. & Fabaceae & & & $\mathrm{x}$ \\
\hline 3. Agonandra brasiliensis Miers & Opiliaceae & & & $\mathrm{x}$ \\
\hline 4. Aiouea saligna Meiss. & Lauraceae & $\mathrm{x}$ & $\mathrm{x}$ & $\mathrm{x}$ \\
\hline 5. Alibertia elliptica (Cham.) K. Schum. & Rubiaceae & $\mathrm{x}$ & $\mathrm{x}$ & $\mathrm{x}$ \\
\hline 6. Amaioua guianensis Aubl. & Rubiaceae & $\mathrm{x}$ & & \\
\hline $\begin{array}{l}\text { 7. Anadenanthera colubrina (Vell.) Brenan } \\
\text { var. cebil (Griseb.) Altschul }\end{array}$ & Mimosaceae & & $\mathrm{x}$ & \\
\hline 8. Andira vermifuga Mart. ex Benth. & Fabaceae & $\mathrm{x}$ & & \\
\hline 9. Apeiba tibourbou Aubl. & Tiliaceae & & & $\mathrm{x}$ \\
\hline 10. Apocynaceae - indet. & Apocynaceae & $\mathrm{x}$ & & \\
\hline $\begin{array}{l}\text { 11. Apuleia leiocarpa (Vog.) Macbr. var. } \\
\text { molaris (Spruce ex Benth.) Koeppen }\end{array}$ & Caesalpiniaceae & $\mathrm{x}$ & $\mathrm{x}$ & $\mathrm{x}$ \\
\hline 12. Arrabidaea cf. brachypoda (DC.) Bur. & Bignoniaceae & & $\mathrm{x}$ & \\
\hline 13. Aspidosperma macrocarpon Mart. & Apocynaceae & & $\mathrm{x}$ & \\
\hline 14. A. subincanum Mart. & Apocynaceae & $\mathrm{x}$ & $\mathrm{x}$ & $\mathrm{x}$ \\
\hline 15. A. tomentosum Mart. & Apocynaceae & & & $\mathrm{x}$ \\
\hline 16. Astrocaryum vulgare Mart. & Arecaceae & $\mathrm{x}$ & $\mathrm{x}$ & $\mathrm{x}$ \\
\hline 17. Astronium fraxinifolium Schott. & Anacardiaceae & $\mathrm{x}$ & $\mathrm{x}$ & $\mathrm{x}$ \\
\hline 18. Bauhinia longifolia (Bongard) Steud. & Caesalpiniaceae & & $\mathrm{x}$ & $\mathrm{x}$ \\
\hline 19. B. outimouta Aubl. & Caesalpiniaceae & $\mathrm{x}$ & $\mathrm{x}$ & $\mathrm{x}$ \\
\hline 20. Bauhinia sp. & Caesalpiniaceae & & & $\mathrm{x}$ \\
\hline 21. Bowdichia virgilioides Kunth & Fabaceae & & $\mathrm{x}$ & \\
\hline 22. Byrsonima laxiflora Griseb. & Malpighiaceae & $\mathrm{x}$ & & $\mathrm{x}$ \\
\hline $\begin{array}{l}\text { 23. Callisthene fasciculata (C.K. Spreng.) } \\
\text { Mart. }\end{array}$ & Vochysiaceae & $\mathrm{x}$ & & \\
\hline 24. Calophyllum brasiliense Cambess. & Clusiaceae & $\mathrm{x}$ & $\mathrm{x}$ & $\mathrm{x}$ \\
\hline 25. Campomanesia eugenioides Blume & Myrtaceae & & $\mathrm{x}$ & \\
\hline 26. Cariniana rubra Gardner ex Miers & Lecythidaceae & & & $\mathrm{x}$ \\
\hline 27. Casearia arborea (L.C. Rich.) Urban & Flacourtiaceae & & $\mathrm{x}$ & $\mathrm{x}$ \\
\hline 28. C. sylvestris $\mathrm{Sw}$. & Flacourtiaceae & & $\mathrm{x}$ & $\mathrm{x}$ \\
\hline 29. Cecropia pachystachya Tréc. & Cecropiaceae & $\mathrm{x}$ & $\mathrm{x}$ & $\mathrm{x}$ \\
\hline $\begin{array}{l}\text { 30. Cheiloclinium cognatum (Miers) } \\
\text { A.C. Smith }\end{array}$ & Hippocrateaceae & $\mathrm{x}$ & $\mathrm{x}$ & \\
\hline 31. Combretum vernicosum Rusby & Combretaceae & & $\mathrm{x}$ & \\
\hline 32. Copaifera langsdorffii Desf. & Caesalpiniaceae & $\mathrm{x}$ & $\mathrm{x}$ & \\
\hline 33. Cordia glabrata (Mart.) A. DC. & Boraginaceae & & $\mathrm{x}$ & \\
\hline 34. C. sellowiana Cham. & Boraginaceae & $\mathrm{x}$ & & $\mathrm{x}$ \\
\hline 35. Coussarea platyphylla Muell. Arg. & Rubiaceae & $\mathrm{x}$ & $\mathrm{x}$ & $\mathrm{x}$ \\
\hline 36. Curatella americana $\mathrm{L}$. & Dilleniaceae & $\mathrm{x}$ & & $\mathrm{x}$ \\
\hline 37. Cuspidaria $\mathrm{sp}$. & Bignoniaceae & & $\mathrm{x}$ & \\
\hline 38. Davilla elliptica St. Hil. & Dilleniaceae & & & $\mathrm{x}$ \\
\hline $\begin{array}{l}\text { 39. Dendropanax cuneatum (DC.) Decne. } \\
\text { \& Planch. }\end{array}$ & Araliaceae & $\mathrm{x}$ & & $\mathrm{x}$ \\
\hline 40. Dilodendron bipinnatum Radlk. & Sapindaceae & & $\mathrm{x}$ & \\
\hline
\end{tabular}


TABLE 1. (Cont'd)

\begin{tabular}{|c|c|c|c|c|}
\hline Species & Family & Upper & Middle & Lower \\
\hline 41. Dioclea cf. glabra Benth. & Fabaceae & $\mathrm{x}$ & & \\
\hline 42. Dioclea sp. & Fabaceae & & $\mathrm{x}$ & \\
\hline 43. Diospyros hispida A. DC. & Ebenaceae & & & $\mathrm{x}$ \\
\hline 44. D. obovata Jacq. & Ebenaceae & $\mathrm{x}$ & $\mathrm{x}$ & \\
\hline 45. D. sericea A. DC. & Ebenaceae & $\mathrm{x}$ & & $\mathrm{x}$ \\
\hline 46. Dipteryx alata Vog. & Fabaceae & & $\mathrm{x}$ & \\
\hline 47. Doliocarpus dentatus (Aubl.) Standl. & Dilleniaceae & $\mathrm{x}$ & & \\
\hline 48. Duguetia marcgraviana Mart. & Annonaceae & $\mathrm{x}$ & $\mathrm{x}$ & $\mathrm{x}$ \\
\hline 49. Emmotum nitens (Benth.) Miers & Icacinaceae & $\mathrm{x}$ & $\mathrm{x}$ & $\mathrm{x}$ \\
\hline 50. Endlicheria paniculata (Spreng.) Macbr. & Lauraceae & & $\mathrm{x}$ & $\mathrm{x}$ \\
\hline $\begin{array}{l}\text { 51. Enterolobium contortisiliquum (Vell.) } \\
\text { Morong }\end{array}$ & Mimosaceae & & $\mathrm{x}$ & \\
\hline 52. Ephedranthus parviflorus S. Moore & Annonaceae & $\mathrm{x}$ & $\mathrm{x}$ & \\
\hline $\begin{array}{l}\text { 53. Eriotheca gracilipes (K. Schum.) } \\
\text { A. Robyns }\end{array}$ & Bombacaceae & & $\mathrm{x}$ & \\
\hline 54. Erythroxylum daphnites Mart. & Erythroxylaceae & & & $\mathrm{x}$ \\
\hline 55. Eugenia aurata Berg & Myrtaceae & & $\mathrm{x}$ & \\
\hline 56. Ficus cf. enormis (Mart. ex Miq.) Miq. & Moraceae & $\mathrm{x}$ & $\mathrm{x}$ & $\mathrm{x}$ \\
\hline 57. Ficus $\mathrm{sp} .1$ & Moraceae & & $\mathrm{x}$ & \\
\hline 58. Ficus sp. 2 & Moraceae & & & $\mathrm{x}$ \\
\hline 59. Genipa americana $\mathrm{L}$. & Rubiaceae & & & $\mathrm{x}$ \\
\hline 60. Guazuma ulmifolia Lam. & Sterculiaceae & & $\mathrm{x}$ & \\
\hline 61. Guettarda viburnioides Cham. \& Schltdl. & Rubiaceae & & $\mathrm{x}$ & \\
\hline 62. Hancornia speciosa Gomez & Apocynaceae & & & $\mathrm{x}$ \\
\hline $\begin{array}{l}\text { 63. Himatanthus bracteatus (A. DC.) } \\
\text { R.E. Woodson }\end{array}$ & Apocynaceae & $\mathrm{x}$ & $\mathrm{x}$ & $\mathrm{x}$ \\
\hline $\begin{array}{l}\text { 64. H. obovatus (Muell. Arg.) } \\
\text { R.E. Woodson }\end{array}$ & Apocynaceae & $\mathrm{x}$ & $\mathrm{x}$ & \\
\hline 65. Hirtella glandulosa Spreng. & Chrysobalanaceae & $\mathrm{x}$ & $\mathrm{x}$ & $\mathrm{x}$ \\
\hline 66. H. gracilipes (Hook.f.) Prance & Chrysobalanaceae & $\mathrm{x}$ & $\mathrm{x}$ & \\
\hline 67. Hieronyma alchorneoides Fr. Allem. & Euphorbiaceae & $\mathrm{x}$ & & $\mathrm{x}$ \\
\hline $\begin{array}{l}\text { 68. Hymenaea courbaril L. var. stilbocarpa } \\
\text { (Hayne) Lee \& Langenheim }\end{array}$ & Caesalpiniaceae & $\mathrm{x}$ & $\mathrm{x}$ & \\
\hline 69. Ilex affinis Gardner & Aquifoliaceae & $\mathrm{x}$ & $\mathrm{x}$ & \\
\hline 70. Inga heterophylla Willd. & Mimosaceae & $\mathrm{x}$ & $\mathrm{x}$ & $\mathrm{x}$ \\
\hline 71. I. thibaudiana DC. & Mimosaceae & $\mathrm{x}$ & $\mathrm{x}$ & $\mathrm{x}$ \\
\hline 72. Jacaranda cuspidifolia Mart. ex A. DC. & Bignoniaceae & & $\mathrm{x}$ & \\
\hline 73. Kielmeyera rubriflora Cambess. & Clusiaceae & & $\mathrm{x}$ & \\
\hline $\begin{array}{l}\text { 74. Licania apetala (E. Meyer) Fritsch var. } \\
\text { apetala }\end{array}$ & Chrysobalanaceae & $\mathrm{x}$ & $\mathrm{x}$ & $\mathrm{x}$ \\
\hline 75. L. blackii Prance & Chrysobalanaceae & $\mathrm{x}$ & $\mathrm{x}$ & $\mathrm{x}$ \\
\hline 76. L. gardneri (Hook.f.) Fritsch & Chrysobalanaceae & $\mathrm{x}$ & & $\mathrm{x}$ \\
\hline 77. Luehea candicans Mart. & Tiliaceae & $\mathrm{x}$ & $\mathrm{x}$ & $\mathrm{x}$ \\
\hline 78. Mabea pohliana (Benth.) Muell. Arg. & Euphorbiaceae & $\mathrm{x}$ & $\mathrm{x}$ & $\mathrm{x}$ \\
\hline 79. Machaerium acutifolium Vog. & Fabaceae & & & $\mathrm{x}$ \\
\hline 80. Magonia pubescens St. Hil. & Sapindaceae & $\mathrm{x}$ & $\mathrm{x}$ & $\mathrm{x}$ \\
\hline
\end{tabular}


TABle 1. (Cont'd)

\begin{tabular}{|c|c|c|c|c|}
\hline Species & Family & Upper & Middle & Lower \\
\hline 81. Matayba guianensis Aubl. & Sapindaceae & $\mathrm{x}$ & & $\mathrm{x}$ \\
\hline 82. Mauritia flexuosa L. & Arecaceae & & & $\mathrm{x}$ \\
\hline 83. Mauritiella armata (Mart.) Burret & Arecaceae & & & $\mathrm{x}$ \\
\hline 84. Maytenus cf. floribunda Reiss. & Celastraceae & & $\mathrm{x}$ & \\
\hline $\begin{array}{l}\text { 85. Micropholis venulosa (Mart. \& Eichl.) } \\
\text { Pierre }\end{array}$ & Sapotaceae & $\mathrm{x}$ & $\mathrm{x}$ & \\
\hline 86. Mimosa laticifera Rizzini \& Mattos & Mimosaceae & $\mathrm{x}$ & $\mathrm{x}$ & $\mathrm{x}$ \\
\hline 87. Moutabea excoriata Mart. ex Miq. & Polygalaceae & $\mathrm{x}$ & & \\
\hline 88. Myrcia amazonica DC. & Myrtaceae & $\mathrm{x}$ & & \\
\hline 89. M. sellowiana Berg & Myrtaceae & $\mathrm{x}$ & $\mathrm{x}$ & $\mathrm{x}$ \\
\hline 90. M. tomentosa (Aubl.) DC. & Myrtaceae & $\mathrm{x}$ & $\mathrm{x}$ & \\
\hline 91. Oenocarpus distichus Mart. & Arecaceae & $\mathrm{x}$ & $\mathrm{x}$ & \\
\hline 92. Ormosia coarctata Jacks. & Fabaceae & $\mathrm{x}$ & & \\
\hline 93. Ouratea castaneaefolia (DC.) Engl. & Ochnaceae & $\mathrm{x}$ & $\mathrm{x}$ & \\
\hline 94. Paragonia pyramidata (L. Rich.) Bureau & Bignoniaceae & & $\mathrm{x}$ & \\
\hline 95. Peltogyne confertiflora (Hayne) Benth. & Caesalpiniaceae & & $\mathrm{x}$ & \\
\hline 96. Physocalymma scaberrimum Pohl & Lythraceae & $\mathrm{x}$ & $\mathrm{x}$ & $\mathrm{x}$ \\
\hline 97. Plathymenia reticulata Benth. & Mimosaceae & & $\mathrm{x}$ & \\
\hline 98. Platypodium elegans Vog. & Fabaceae & & $\mathrm{x}$ & $\mathrm{x}$ \\
\hline 99. Posoqueria aff. macropus Mart. & Rubiaceae & $\mathrm{x}$ & & $\mathrm{x}$ \\
\hline 100. Pouteria cf. macrophylla (Lam.) Eyma & Sapotaceae & $\mathrm{x}$ & $\mathrm{x}$ & $\mathrm{x}$ \\
\hline 101. P. torta (Mart.) Radlk. & Sapotaceae & & $\mathrm{x}$ & \\
\hline 102. Protium heptaphyllum (Aubl.) March. & Burseraceae & $\mathrm{x}$ & $\mathrm{x}$ & $\mathrm{x}$ \\
\hline 103. P. spruceanum (Benth.) Engl. & Burseraceae & $\mathrm{x}$ & $\mathrm{x}$ & $\mathrm{x}$ \\
\hline $\begin{array}{l}\text { 104. Pseudobombax longiflorum (Mart. \& } \\
\text { Zucc.) A. Robyns }\end{array}$ & Bombacaceae & & & $\mathrm{x}$ \\
\hline 105. Pseudolmedia laevigata Tréc. & Moraceae & $\mathrm{x}$ & $\mathrm{x}$ & $\mathrm{x}$ \\
\hline 106. Qualea multiflora Mart. & Vochysiaceae & & $\mathrm{x}$ & $\mathrm{x}$ \\
\hline 107. Rubiaceae - indet. & Rubiaceae & $\mathrm{x}$ & & \\
\hline 108. Salacia elliptica (Mart.) G. Don & Hippocrateaceae & $\mathrm{x}$ & & \\
\hline $\begin{array}{l}\text { 109. Schefflera morototoni (Aubl.) } \\
\text { B. Maguire, Steyerm. \& D.G. Frodin }\end{array}$ & Araliaceae & $\mathrm{x}$ & $\mathrm{x}$ & $\mathrm{x}$ \\
\hline 110. Sclerolobium paniculatum Vog. & Caesalpiniaceae & $\mathrm{x}$ & $\mathrm{x}$ & $\mathrm{x}$ \\
\hline 111. Serjania glutinosa Radlk. & Sapindaceae & & $\mathrm{x}$ & \\
\hline 112. Siparuna guianensis Aubl. & Monimiaceae & $\mathrm{x}$ & $\mathrm{x}$ & $\mathrm{x}$ \\
\hline 113. Sorocea klotzschiana Baill. & Moraceae & & & $\mathrm{x}$ \\
\hline 114. Sterculia excelsa Mart. & Sterculiaceae & $\mathrm{x}$ & $\mathrm{x}$ & $\mathrm{x}$ \\
\hline 115. S. striata St. Hil. \& Naud. & Sterculiaceae & & $\mathrm{x}$ & \\
\hline $\begin{array}{l}\text { 116. Tabebuia impetiginosa (Mart. ex DC.) } \\
\text { Standl. }\end{array}$ & Bignoniaceae & $\mathrm{x}$ & $\mathrm{x}$ & $\mathrm{x}$ \\
\hline 117. Tapirira guianensis Aubl. & Anacardiaceae & $\mathrm{x}$ & $\mathrm{x}$ & $\mathrm{x}$ \\
\hline 118. Tapura amazonica Poepp. \& Endl. & Dichapetalaceae & & & $\mathrm{x}$ \\
\hline 119. Tetragastris altissima (Aubl.) Swart. & Burseraceae & $\mathrm{x}$ & $\mathrm{x}$ & $\mathrm{x}$ \\
\hline 120. Tetrapterys glabra (Spreng.) Griseb. & Malpighiaceae & $\mathrm{x}$ & & \\
\hline 121. Tococa formicaria Mart. & Melastomataceae & $\mathrm{x}$ & & $\mathrm{x}$ \\
\hline 122. Unonopsis lindmanii R.E. Fries & Annonaceae & & $\mathrm{x}$ & \\
\hline
\end{tabular}


TABle 1. (Cont'd)

\begin{tabular}{|c|c|c|c|c|c|c|c|}
\hline \multicolumn{4}{|l|}{ Species } & Family & Upper & Middle & Lower \\
\hline \multirow{2}{*}{\multicolumn{4}{|c|}{$\begin{array}{l}\text { 123. Virola urbaniana Warb. } \\
\text { 124. Vismia sp. }\end{array}$}} & Myristicaceae & $\mathrm{x}$ & $\mathrm{x}$ & $\mathrm{x}$ \\
\hline & & & & Clusiaceae & & & $\mathrm{x}$ \\
\hline \multicolumn{4}{|c|}{ 125. Vitex polygama Cham. } & Verbenaceae & $\mathrm{x}$ & $\mathrm{x}$ & $\mathrm{x}$ \\
\hline \multicolumn{4}{|c|}{$\begin{array}{l}\text { 126. Vochysia haenkeana Mart. } \\
\text { 127. Xylopia aromatica (Lam.) Mart. }\end{array}$} & Vochysiaceae & & $\mathrm{x}$ & \\
\hline \multicolumn{4}{|c|}{$\begin{array}{l}\text { 127. Xylopia aromatica (Lam.) Mart. } \\
\text { 128. X. emarginata Mart. }\end{array}$} & Annonaceae & $\mathrm{x}$ & $\mathrm{x}$ & $\mathrm{x}$ \\
\hline \multirow{2}{*}{\multicolumn{4}{|c|}{$\begin{array}{l}\text { 128. X. emarginata Mart. } \\
\text { 129. Zanthoxylum riedelianum Engl. }\end{array}$}} & Annonaceae & $\mathrm{x}$ & $\mathrm{x}$ & \\
\hline & & & & Rutaceae & & $\mathrm{x}$ & $\mathrm{x}$ \\
\hline Total & Upper & Middle & Lower & & & & \\
\hline No. of species & 74 & 86 & 77 & & & & \\
\hline No. of genera & 62 & 73 & 67 & & & & \\
\hline No. of families & 37 & 38 & 41 & & & & \\
\hline
\end{tabular}

TABLE 2. Simpson's and Shannon's diversity indices and evenness in upper, middle and lower sections of the Bacaba gallery forest, and total area sampled

\begin{tabular}{llll}
\hline \hline & & & $\begin{array}{l}\text { Pielou's evenness index } \\
\left(\mathrm{J}^{\prime}=\mathrm{H}^{\prime} / \ln (S)\right)\end{array}$ \\
\hline Upper & 3.84 & 34.57 & 0.89 \\
Middle & 4.08 & 49.09 & 0.91 \\
Lower & 3.57 & 24.03 & 0.82 \\
Total area & 4.21 & 48.32 & 0.87 \\
\hline \hline
\end{tabular}

Ephedranthus parviflorus (11.5), which together represented $21.3 \%$ of the total IVI. Hymenaea courbaril var. stilbocarpa, with only nine large individuals, occupied the first IVI position. Relative dominance $(\mathrm{RDo}=11.3 \%)$ was the most important component of the IVI of this species, while density was the most important for $T$. altissima, the second species in IVI, with RD of $4.9 \%$. Eighteen species were represented by single individuals. Tetragastris altissima and Aspidosperma subincanum were the most constant species in the sampling, each with RF of $4.0 \%$. Only eight species occurred in $20 \%$ or more of the plots, while 21 occurred only in single plots.

The average heights of the most important species were above $15 \mathrm{~m}$, and some emergent individuals of $H$. courbaril var. stilbocarpa, A. leiocarpa and P. torta reached $25 \mathrm{~m}$. All individuals of E. parviflorus were under $10 \mathrm{~m}$ high. Alibertia elliptica and Luehea candicans were restricted to the understorey, with the tallest individuals under $5 \mathrm{~m}$.

\section{Lower section}

The absolute density was 1351 individuals/ha and basal area $23.46 \mathrm{~m}^{2} /$ ha. Dead individuals represented $6.93 \%$ of the total density and $3.51 \%$ of the basal area. The 
TABLE 3. Phytosociological indices for species in the upper section plots of the Bacaba gallery forest. The $27 \mathrm{spp}$. listed constitute $75 \%$ of total IVI (226 units). A further 48 spp. account for the other $25 \%$ of IVI. AD, absolute density (individuals/ha); RD, relative density (\%); ADo, absolute dominance (basal area/ha); RDo, relative dominance (\%); AF, absolute frequency ( $\%$ of plots at which a species occurs); RF, relative frequency - (frequency of a species/sum frequency of all species) $\times 100$. Total density: 1023 individuals/ha; total basal area: $20.44 \mathrm{~m}^{2} /$ ha; total sampling area: $4700 \mathrm{~m}^{2}$

\begin{tabular}{|c|c|c|c|c|c|c|c|c|}
\hline \multirow[b]{2}{*}{ Species } & & \multicolumn{2}{|c|}{ Density } & \multicolumn{2}{|c|}{ Dominance } & \multicolumn{2}{|c|}{ Frequency } & \multirow[b]{2}{*}{ IVI } \\
\hline & & $\mathrm{AD}$ & $\mathrm{RD}$ & ADo & RDo & $\mathrm{AF}$ & $\mathrm{RF}$ & \\
\hline 1. & Diospyros obovata & 100.0 & 9.77 & 1.484 & 7.26 & 44.68 & 5.90 & 22.93 \\
\hline 2. & Dead individuals & 51.1 & 4.99 & 0.836 & 4.09 & 34.04 & 4.49 & 13.57 \\
\hline 3. & Calophyllum brasiliense & 27.7 & 2.70 & 1.531 & 7.49 & 17.02 & 2.25 & 12.44 \\
\hline 4. & Tetragastris altissima & 44.7 & 4.37 & 0.670 & 3.28 & 36.17 & 4.78 & 12.42 \\
\hline 5. & Protium heptaphyllum & 40.4 & 3.95 & 0.724 & 3.54 & 31.91 & 4.21 & 11.71 \\
\hline 6. & Astrocaryum vulgare & 51.1 & 4.99 & 0.405 & 1.98 & 29.79 & 3.93 & 10.90 \\
\hline 7. & Apuleia leiocarpa & 23.4 & 2.29 & 1.271 & 6.22 & 17.02 & 2.25 & 10.75 \\
\hline 8. & Mabea pohliana & 42.6 & 4.16 & 0.456 & 2.23 & 27.66 & 3.65 & 10.04 \\
\hline 9. & $\begin{array}{l}\text { Physocalymma } \\
\text { scaberrimum }\end{array}$ & 23.4 & 2.29 & 0.809 & 3.96 & 23.40 & 3.09 & 9.34 \\
\hline 10. & Licania blackii & 31.9 & 3.12 & 0.585 & 2.86 & 23.40 & 3.09 & 9.06 \\
\hline 11. & $\begin{array}{l}\text { Hymenaea courbaril var. } \\
\text { stilbocarpa }\end{array}$ & 10.6 & 1.04 & 1.288 & 6.30 & 10.64 & 1.40 & 8.74 \\
\hline 12. & Oenocarpus distichus & 27.7 & 2.70 & 0.566 & 2.77 & 21.28 & 2.81 & 8.28 \\
\hline 13. & Tapirira guianensis & 14.9 & 1.46 & 0.977 & 4.78 & 14.89 & 1.97 & 8.20 \\
\hline 14. & Himatanthus bracteatus & 27.7 & 2.70 & 0.421 & 2.06 & 17.02 & 2.25 & 7.01 \\
\hline 15. & Aspidosperma subincanum & 27.7 & 2.70 & 0.354 & 1.73 & 19.15 & 2.53 & 6.97 \\
\hline 16. & Protium spruceanum & 10.6 & 1.04 & 0.850 & 4.16 & 8.51 & 1.12 & 6.32 \\
\hline 17. & Licania apetala & 23.4 & 2.29 & 0.337 & 1.65 & 17.02 & 2.25 & 6.18 \\
\hline 18. & Ephedranthus parviflorus & 27.7 & 2.70 & 0.247 & 1.21 & 14.89 & 1.97 & 5.88 \\
\hline 19. & Bauhinia outimouta & 21.3 & 2.08 & 0.260 & 1.27 & 19.15 & 2.53 & 5.87 \\
\hline 20. & Myrcia sellowiana & 19.1 & 1.87 & 0.298 & 1.46 & 17.02 & 2.25 & 5.57 \\
\hline 21. & Ormosia coarctata & 23.4 & 2.29 & 0.237 & 1.16 & 14.89 & 1.97 & 5.42 \\
\hline 22. & Vitex polygama & 10.6 & 1.04 & 0.544 & 2.66 & 10.64 & 1.40 & 5.10 \\
\hline 23. & Pseudolmedia laevigata & 19.1 & 1.87 & 0.239 & 1.17 & 14.89 & 1.97 & 5.00 \\
\hline 24. & Inga thibaudiana & 19.1 & 1.87 & 0.245 & 1.20 & 12.77 & 1.69 & 4.76 \\
\hline 25. & Licania gardneri & 14.9 & 1.46 & 0.268 & 1.31 & 14.89 & 1.97 & 4.74 \\
\hline 26. & Luehea candicans & 14.9 & 1.46 & 0.219 & 1.07 & 14.89 & 1.97 & 4.49 \\
\hline 27. & Andira vermifuga & 6.4 & 0.62 & 0.554 & 2.71 & 6.38 & 0.84 & 4.18 \\
\hline $28-75$. & 48 other spp. & 267.6 & 26.18 & 3.765 & 18.42 & 223.39 & 29.47 & 74.13 \\
\hline Total & & 1023 & 100 & 20.44 & 100 & 757.4 & 100 & 300 \\
\hline
\end{tabular}

Estimate of the confidence interval $(\mathrm{CI})$ :

Density: $\mathrm{CI}=\mathrm{P}[869 \leq \mu \leq 1177]=0.95$; basal area: $\mathrm{CI}=\mathrm{P}[17.24 \leq \mu \leq 23.62]=0.95$.

highest importance values (IVI) (Table 5) were recorded for Mauritia flexuosa (50.5), Cecropia pachystachya (24.1), Astrocaryum vulgare (18.2), Tapirira guianensis (16.5) and Xylopia aromatica (13.7), which together represented $41 \%$ of the total IVI. 
TABLE 4. Phytosociological indices for species in the middle section plots of the Bacaba gallery forest. The $34 \mathrm{spp}$. listed constitute $75.7 \%$ of total IVI (227 units). A further $53 \mathrm{spp}$. account for the other $24.3 \%$ of IVI. AD, absolute density (individuals/ha); RD, relative density $(\%)$; ADo, absolute dominance (basal area/ha); RDo, relative dominance (\%); AF, absolute frequency ( $\%$ of plots at which a species occurs); RF, relative frequency - (frequency of a species/sum frequency of all species) $\times 100$. Total density: 962 individuals/ha; total basal area: $22.28 \mathrm{~m}^{2} /$ ha; total sampling area: $4700 \mathrm{~m}^{2}$

\begin{tabular}{|c|c|c|c|c|c|c|c|c|}
\hline \multirow[b]{2}{*}{ Species } & & \multicolumn{2}{|c|}{ Density } & \multicolumn{2}{|c|}{ Dominance } & \multicolumn{2}{|c|}{ Frequency } & \multirow[b]{2}{*}{ IVI } \\
\hline & & $\mathrm{AD}$ & $\mathrm{RD}$ & ADo & RDo & $\mathrm{AF}$ & $\mathrm{RF}$ & \\
\hline 1. & $\begin{array}{l}\text { Hymenaea courbaril var. } \\
\text { stilbocarpa }\end{array}$ & 19.1 & 1.99 & 2.520 & 11.31 & 14.89 & 2.01 & 15.31 \\
\hline 2. & Tetragastris altissima & 46.8 & 4.87 & 0.898 & 4.03 & 29.79 & 4.02 & 12.92 \\
\hline 3. & Apuleia leiocarpa & 21.3 & 2.21 & 1.615 & 7.25 & 21.28 & 2.87 & 12.34 \\
\hline 4. & Pouteria torta & 12.8 & 1.33 & 1.956 & 8.78 & 12.77 & 1.72 & 11.83 \\
\hline 5. & Ephedranthus parviflorus & 53.2 & 5.53 & 0.504 & 2.26 & 27.66 & 3.74 & 11.52 \\
\hline 6. & $\begin{array}{l}\text { Aspidosperma } \\
\text { subincanum }\end{array}$ & 42.6 & 4.42 & 0.682 & 3.06 & 29.79 & 4.02 & 11.51 \\
\hline 7. & Vitex polygama & 8.5 & 0.88 & 1.658 & 7.44 & 8.51 & 1.15 & 9.48 \\
\hline 8. & Luehea candicans & 36.2 & 3.76 & 0.301 & 1.35 & 27.66 & 3.74 & 8.85 \\
\hline 9. & Protium heptaphyllum & 42.6 & 4.42 & 0.276 & 1.24 & 23.40 & 3.16 & 8.82 \\
\hline 10. & Dead individuals & 23.4 & 2.43 & 0.633 & 2.84 & 23.40 & 3.16 & 8.44 \\
\hline 11. & Pouteria cf. macrophylla & 29.8 & 3.10 & 0.441 & 1.98 & 21.28 & 2.87 & 7.95 \\
\hline 12. & Sclerolobium paniculatum & 27.7 & 2.88 & 0.602 & 2.70 & 17.02 & 2.30 & 7.88 \\
\hline 13. & Protium spruceanum & 25.5 & 2.65 & 0.479 & 2.15 & 14.89 & 2.01 & 6.82 \\
\hline 14. & Unonopsis lindmanii & 27.7 & 2.88 & 0.214 & 0.96 & 12.77 & 1.72 & 5.56 \\
\hline 15. & Alibertia elliptica & 21.3 & 2.21 & 0.156 & 0.70 & 19.15 & 2.59 & 5.50 \\
\hline 16. & Calophyllum brasiliense & 6.4 & 0.66 & 0.858 & 3.85 & 6.38 & 0.86 & 5.37 \\
\hline 17. & Diospyros obovata & 14.9 & 1.55 & 0.457 & 2.05 & 10.64 & 1.44 & 5.03 \\
\hline 18. & Tabebuia impetiginosa & 6.4 & 0.66 & 0.769 & 3.45 & 6.38 & 0.86 & 4.98 \\
\hline 19. & Mabea pohliana & 21.3 & 2.21 & 0.102 & 0.46 & 17.02 & 2.30 & 4.97 \\
\hline 20. & Copaifera langsdorffii & 4.3 & 0.44 & 0.878 & 3.94 & 4.26 & 0.57 & 4.96 \\
\hline 21. & Cecropia pachystachya & 17.0 & 1.77 & 0.345 & 1.55 & 10.64 & 1.44 & 4.75 \\
\hline 22. & Platypodium elegans & 17.0 & 1.77 & 0.174 & 0.78 & 14.89 & 2.01 & 4.56 \\
\hline 23. & $\begin{array}{l}\text { Anadenanthera colubrina } \\
\text { var. cebil }\end{array}$ & 10.6 & 1.11 & 0.428 & 1.92 & 10.64 & 1.44 & 4.47 \\
\hline 24. & Sterculia striata & 6.4 & 0.66 & 0.648 & 2.91 & 6.38 & 0.86 & 4.44 \\
\hline 25. & Aiouea saligna & 17.0 & 1.77 & 0.140 & 0.63 & 14.89 & 2.01 & 4.41 \\
\hline 26. & Siparuna guianensis & 23.4 & 2.43 & 0.067 & 0.30 & 10.64 & 1.44 & 4.17 \\
\hline 27. & Astronium fraxinifolium & 12.8 & 1.33 & 0.241 & 1.08 & 12.77 & 1.72 & 4.13 \\
\hline 28. & Coussarea platyphylla & 19.1 & 1.99 & 0.145 & 0.65 & 10.64 & 1.44 & 4.07 \\
\hline 29. & $\begin{array}{l}\text { Physocalymma } \\
\text { scaberrimum }\end{array}$ & 12.8 & 1.33 & 0.189 & 0.85 & 12.77 & 1.72 & 3.90 \\
\hline 30. & Pseudolmedia laevigata & 17.0 & 1.77 & 0.152 & 0.68 & 10.64 & 1.44 & 3.89 \\
\hline 31. & Combretum vernicosum & 12.8 & 1.33 & 0.123 & 0.55 & 12.77 & 1.72 & 3.60 \\
\hline 32. & Oenocarpus distichus & 10.6 & 1.11 & 0.301 & 1.35 & 8.51 & 1.15 & 3.60 \\
\hline 33. & Inga thibaudiana & 12.8 & 1.33 & 0.247 & 1.11 & 8.51 & 1.15 & 3.59 \\
\hline 34. & Xylopia aromatica & 14.9 & 1.55 & 0.082 & 0.37 & 10.64 & 1.44 & 3.36 \\
\hline $35-87$. & 53 other spp. & 266.0 & 27.67 & 2.999 & 13.47 & 236.13 & 31.91 & 73.02 \\
\hline Total & & 962 & 100 & 22.28 & 100 & 740.4 & 100 & 300 \\
\hline
\end{tabular}

Estimate of the confidence interval $(\mathrm{CI})$ :

Density: $\mathrm{CI}=\mathrm{P}[837 \leq \mu \leq 1085]=0.95$; basal area: $\mathrm{CI}=\mathrm{P}[20.35 \leq \mu \leq 23.77]=0.95$. 
TABLE 5. Phytosociological indices for species in the lower section plots of the Bacaba gallery forest. The $20 \mathrm{spp}$. listed constitute $75 \%$ of total IVI (225 units). A further $58 \mathrm{spp}$. account for the other $25 \%$ of IVI. AD, absolute density (individuals/ha); RD, relative density (\%); ADo, absolute dominance (basal area/ha); RDo, relative dominance (\%); AF, absolute frequency ( $\%$ of plots at which a species occurs); RF, relative frequency - (frequency of a species/sum frequency of all species) $\times 100$. Total density: 1351 individuals/ha; total basal area: $23.46 \mathrm{~m}^{2} /$ ha; total sampling area: $4700 \mathrm{~m}^{2}$

\begin{tabular}{|c|c|c|c|c|c|c|c|c|}
\hline \multirow[b]{2}{*}{ Species } & & \multicolumn{2}{|c|}{ Density } & \multicolumn{2}{|c|}{ Dominance } & \multicolumn{2}{|c|}{ Frequency } & \multirow[b]{2}{*}{ IVI } \\
\hline & & $\mathrm{AD}$ & $\mathrm{RD}$ & ADo & RDo & $\mathrm{AF}$ & $\mathrm{RF}$ & \\
\hline 1. & Mauritia flexuosa & 87.2 & 6.46 & 9.188 & 39.16 & 40.43 & 4.88 & 50.50 \\
\hline 2. & Cecropia pachystachya & 125.5 & 9.29 & 1.659 & 7.07 & 63.83 & 7.71 & 24.07 \\
\hline 3. & Astrocaryum vulgare & 110.6 & 8.19 & 1.201 & 5.12 & 40.43 & 4.88 & 18.20 \\
\hline 4. & Dead individuals & 93.6 & 6.93 & 0.824 & 3.51 & 53.19 & 6.43 & 16.86 \\
\hline 5. & Tapirira guianensis & 72.3 & 5.35 & 1.356 & 5.78 & 44.68 & 5.40 & 16.53 \\
\hline 6. & Xylopia aromatica & 76.6 & 5.67 & 0.751 & 3.20 & 40.43 & 4.88 & 13.75 \\
\hline 7. & $\begin{array}{l}\text { Physocalymma } \\
\text { scaberrimum }\end{array}$ & 55.3 & 4.09 & 0.786 & 3.35 & 40.43 & 4.88 & 12.33 \\
\hline 8. & Virola urbaniana & 83.0 & 6.14 & 0.493 & 2.10 & 31.91 & 3.86 & 12.10 \\
\hline 9. & Mabea pohliana & 44.7 & 3.31 & 0.263 & 1.12 & 31.91 & 3.86 & 8.28 \\
\hline 10. & Cordia sellowiana & 36.2 & 2.68 & 0.488 & 2.08 & 23.40 & 2.83 & 7.58 \\
\hline 11. & Himatanthus bracteatus & 42.6 & 3.15 & 0.364 & 1.55 & 23.40 & 2.83 & 7.53 \\
\hline 12. & Inga thibaudiana & 34.0 & 2.52 & 0.591 & 2.52 & 19.15 & 2.31 & 7.36 \\
\hline 13. & Aspidosperma subincanum & 38.3 & 2.83 & 0.352 & 1.50 & 19.15 & 2.31 & 6.65 \\
\hline 14. & Endlicheria paniculata & 31.9 & 2.36 & 0.277 & 1.18 & 23.40 & 2.83 & 6.37 \\
\hline 15. & Acosmium sp. & 27.7 & 2.05 & 0.523 & 2.23 & 17.02 & 2.06 & 6.34 \\
\hline 16. & Bauhinia longifolia & 19.1 & 1.42 & 0.143 & 0.61 & 17.02 & 2.06 & 4.08 \\
\hline 17. & Tetragastris altissima & 17.0 & 1.26 & 0.397 & 1.69 & 8.51 & 1.03 & 3.98 \\
\hline 18. & Coussarea platyphylla & 19.1 & 1.42 & 0.223 & 0.95 & 12.77 & 1.54 & 3.91 \\
\hline 19. & Vismia sp. & 17.0 & 1.26 & 0.127 & 0.54 & 14.89 & 1.80 & 3.60 \\
\hline 20. & Sterculia excelsa & 10.6 & 0.79 & 0.303 & 1.29 & 10.64 & 1.29 & 3.36 \\
\hline $21-78$. & 58 other spp. & 308.7 & 22.83 & 3.151 & 13.45 & 251.11 & 30.33 & 66.62 \\
\hline Total & & 1351 & 100 & 23.46 & 100 & 827.7 & 100 & 300 \\
\hline
\end{tabular}

Estimate of the confidence interval $(\mathrm{CI})$ :

Density: $C I=P[1213 \leq \mu \leq 1467]=0.95$; basal area: $C I=P[19.84 \leq \mu \leq 27]=0.95$.

For M. flexuosa, relative dominance $(\mathrm{RDo}=39 \%)$ was the most important component of the IVI composition. Twenty species were represented by only one individual. Cecropia pachystachya $(\mathrm{RF}=7.71 \%)$ and $T$. guianensis $(\mathrm{RF}=5.4 \%)$ were the most constant species in the area. Twelve species occurred in $20 \%$ or more of the plots, while 24 occurred only in single plots.

Only M. flexuosa stood out as an emergent, reaching $25 \mathrm{~m}$, while some individuals of Physocalymma scaberrimum and T. guianensis reached $20 \mathrm{~m}$. All individuals of C. pachystachya and Astrocaryum vulgare were under 10m and the Acosmium sp. was restricted to the understorey, with heights under $5 \mathrm{~m}$. 


\section{Similarity}

The Venn diagram (Zar, 1999) in Fig. 3 shows the floristic relationships of the sections. Sørensen's coefficients of similarity gave the same relatively high figure of 0.65 for both the comparisons of the middle section with the upper and the upper with the lower, but a rather smaller value of 0.56 for comparison of the middle with the lower. The Morisita index, which takes species importance into account in addition to occurrence, agreed with Sørensen's in its high value $(\mathrm{C} \lambda=0.66)$ for comparison of upper with middle sections, but this fell to $\mathrm{C} \lambda=0.41$ for upper with lower and to $\mathrm{C} \lambda=0.36$ for middle with lower.

\section{DiscusSiON}

The flora of the Bacaba forest is probably characteristic of galleries in Eastern Mato Grosso, judging by the few other detailed surveys which have been conducted (Ratter et al., 1973; Felfili et al., 1998). The vast majority of the species are widespread in Central Brazil, and amongst the most common are Hymenaea courbaril var. stilbocarpa, Copaifera langsdorffii, Apuleia leiocarpa, Siparuna guianensis, Calophyllum brasiliense, Cariniana rubra, Protium heptaphyllum, Tapirira guianensis, Matayba guianensis and the weedy colonizers Sclerolobium paniculatum and Cecropia pachystachya. Ten of the 13 species quoted by Oliveira-Filho \& Ratter (1995) as being the most frequent in 13 gallery forests were found in the Bacaba.

A number of elements differing in habitat preference are found amongst the species listed in Table 1. Species of wetter and swampy habitats are represented by, for instance, Calophyllum brasiliense, Dendropanax cuneatum, Hieronyma alchorneoides, Mauritia flexuosa, Mauritiella armata, Oenocarpus distichus, Pseudolmedia laevigata and Xylopia emarginata. Those typical of the mesophilous forests of mesotrophic

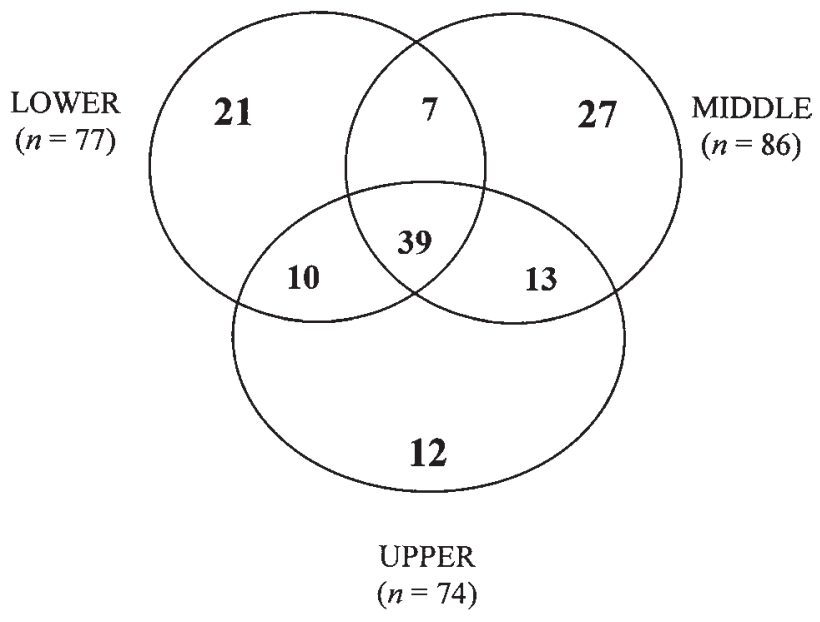

FIG. 3. Venn diagram showing floristic relationships of the sections (species shared and exclusive). 
soils are represented by Anadenanthera colubrina var. cebil, Aspidosperma subincanum, Cordia glabrata, Copaifera langsdorffi, Enterolobium contortisiliquum, Platypodium elegans, Sterculia striata, Tabebuia impetiginosa, Vitex polygama and Zanthoxylum riedelianum. The cerrado element is represented by many species such as Aspidosperma macrocarpon, A. tomentosum, Bowdichia virgilioides, Curatella americana, Davilla elliptica, Himatanthus obovatus, Kielmeyera rubriflora, Qualea multiflora, etc., while Emmotum nitens and Hirtella glandulosa are typical of the dystrophic element of cerradão and Callisthene fasciculata, Dipteryx alata, Luehea candicans and Magonia pubescens of the mesotrophic (Ratter, 1971; Ratter et al., 1973, 1977). Colonizing species, often particularly associated with human disturbance, are also common, for example Apeiba tibourbou, Astrocaryum vulgare, Cecropia pachystachya, Genipa americana, Sclerolobium paniculatum and Xylopia aromatica. The species list reflects the variety of microhabitats and other environmental factors of the Bacaba gallery and the ecotones occurring there.

Ratter et al. (1973) suggested that the gallery forests of the Eastern Mato Grosso contain many elements of the Amazonian forest flora since the rivers on which they occur are part of the Amazon drainage. Oliveira-Filho \& Ratter (1995), studying the phytogeography of South American forests, also found strong floristic links between these gallery forests and the Amazonian flora. By contrast, the gallery and valley forests of the Chapada dos Guimarães in central-southern Mato Grosso have stronger links with the Atlantic forests, probably because of the climatic conditions dictated by the high altitude and their location in the Paraná-Paraguai River basin (Pinto \& Oliveira-Filho, 1999). In the present study Oenocarpus distichus and Astrocaryum vulgare are examples of typical Amazonian palms (the vernacular name of the former is 'Bacaba' and it gives its name to the stream). Tapura amazonica, Protium spruceanum, Hirtella glandulosa and Vochysia haenkeana are examples of widespread species that extend their distribution to the Amazon basin (OliveiraFilho \& Ratter, 1995), as do many others, for example Abuta grandifolia, Moutabea excoriata, Schefflera morototoni, Sterculia excelsa and those species originally described from Guyana, Amaioua guianensis, Matayba guianensis, Siparuna guianensis and Tapirira guianensis.

Shannon's diversity index was high for all sections, ranging from 3.84 nats/individual in the lower section to 4.08 in the middle. This index is sensitive to species richness and to evenness, normally ranging from 1.5 to 3.5 (Magurran, 1988). Evenness was higher in the upper section than in the lower, even though the latter was richer in species. Values of Shannon's index found in other studies in gallery forests in Mato Grosso (Oliveira-Filho, 1989; Pinto \& Oliveira-Filho, 1999) were in the same range as those found in this study, even though different methodologies were used. Simpson's index (1/Ds), which gives more weight to the common species (Magurran, 1988), and evenness were lower in the lower portion as a result of the dominance of Mauritia flexuosa.

Density was slightly higher in the lower wetter portion (Table 5), agreeing with other descriptions of seasonally flooded sites in gallery forests (Walter, 1995). The 
dominance of palms such as Mauritia flexuosa is typical of such flooded sites. Astrocaryum vulgare, another palm, occurred in the lower section but also in welldrained upper section sites which are occasionally subjected to disturbances by fire. Lorenzi (1996) also described the occurrence of this species in both conditions. The higher percentage of dead standing individuals in the lower and upper sections, compared with the middle, is also an indication of disturbances in both these sections.

The species with highest IVI differed between the sections, and only Tetragastris altissima had high IVI in both upper and middle sections. It appears to be a species well adapted to a steep relief with rock outcrops.

\section{Conclusion}

The Bacaba forest has many species in common with other gallery forests of the cerrado biome and shows strong floristic links with the Amazonian (Hylaean) forest. This reflects its position in the ecotonal zone between the cerrado and Amazonian forest biomes in the Mortes-Araguaia-Amazon basin.

Occurrence and relative importance of species in the vegetation mosaic sampled in the plots are related to environmental conditions, particularly humidity gradients.

\section{ACKNOWLEDGEMENTS}

We are grateful to the Foundation to Support Research in Mato Grosso State (FAPEMAT) for the grant to carry out this research (contract no. 2.01.00055/1998-05). Several botanists provided species identifications, especially Dr James Alexander Ratter. Claudonei das Neves Rosa, Adão Luiz Patrocínio, Adair José Rodrigues, Wânia Maria Gonçalves Duarte and Juvenal Pinheiro Neto assisted in the field work. Mr Christopher William Fagg and Dr Ratter kindly reviewed the manuscript.

\section{REFERENCES}

Brinson, M. M. (1990). Riverine forest. In: Lugo, A. E., Brinson, M. M. \& Brown, S. (eds) Ecosystems of the World 15 - Forested Wetlands, pp. 87-141. Amsterdam: Elsevier Publishers.

Brower, J. E. \& ZAR, J. H. (1977). Field and Laboratory Methods for General Ecology. Iowa: W. C. Brown Co.

Camargo, A. P. (1963). Clima do cerrado. In: Ferri, M. G. (co-ordinator) Simpósio Sobre o Cerrado, pp. 75-95. São Paulo: EDUSP.

Cochrane, T. T., Sánchez, L. G. A., Porras, J. A. \& Garver, C. L. (1985). Land in Tropical America, Vol. 3. Cali: CIAT/EMBRAPA-CPAC,

Curtis, J. T. \& MCIntosh, R. P. (1950). The interrelations of certain analytic and synthetic phytosociological characters. Ecology 31: 434-455.

Curtis, J. T. \& McIntosh, R. P. (1951). An upland forest continuum in the prairieforest border region of Wisconsin. Ecology 32: 476-496.

Felfili, J. M. (1995). Diversity, structure and dynamics of a gallery forest in central Brazil. Vegetatio 117: 1-15.

Felfili, J. M. (1997). Dynamics of the natural regeneration in the Gama gallery forest in central Brazil. Forest Ecol. Manag. 91: 235-245. 
Felfili, J. M., Silva-Júnior, M. C. \& Nogueira, P. E. (1998). Levantamento da vegetação arbórea na região de Nova Xavantina, MT. Bol. Herb. Ezechias Paulo Heringer 3: 63-81.

Kellman, M. \& Meave, J. (1997). Fire in the tropical gallery forests of Belize. J. Biogeogr. 24: 23-24.

Lorenzi, H. (1996). Palmeiras no Brasil: nativas e exóticas. Nova Odessa, Brazil: Editora Plantarum Ltda.

Magurran, E. (1988). Ecological Diversity and its Measurement. London: Croom Helm.

Marimon, B. S., Varella, R. F. \& Marimon-Júnior, B. H. (1998).

Fitossociologia de uma área de cerrado de encosta em Nova Xavantina, Mato Grosso. Bol. Herb. Ezechias Paulo Heringer 3: 82-101.

Oliveira-Filho, A. T. (1989). Composição florística e estrutura comunitária da floresta de galeria do Córrego da Paciência, Cuiabá (MT). Acta Bot. Brasil 3: 91-112.

Oliveira-Filho, A. T. \& Ratter, J. A. (1995). A study of the origin of central Brazilian forests by the analysis of plant species distribution patterns. Edinb. J. Bot. 52: 141-194.

Oliveira-Filho, A. T., Scolforo, J. R. \& Mello, J. M. (1994a). Composição florística e estrutura comunitária de um remanescente de floresta semidecídua montana em Lavras (MG). Revista Brasil. Bot. 17: 169-184.

Oliveira-Filho, A. T., Vilela, E. A., Carvalho, D. A. \& Gavilanes, M. L. (1994b). Effects of soils and topography on the distribution of tree species in a tropical riverine forest in south-eastern Brazil. J. Trop. Ecol. 10: 483-508.

Philip, M. S. (1994). Measuring Trees and Forests, 2nd edition. Cambridge: Cambridge University Press.

Pinto, J. R. R. \& Oliveira-Filho, A. T. (1999). Perfil florístico e estrutura da comunidade arbórea de uma floresta de vale no Parque Nacional da Chapada dos Guimarães, Mato Grosso, Brasil. Revista Brasil. Bot. 22: 53-67.

RATteR, J. A. (1971). Some notes on two types of cerradão occurring in northeastern Mato Grosso. In: Fer ri, M. G. (ed.) III Simpósio Sobre o Cerrado, pp. 100-102. São Paulo: EDUSP.

Ratter, J. A., Richards, P. W., Argent, G. \& Gifford, D. R. (1973). Observations on the vegetation of northeastern Mato Grosso. I. The woody vegetation types of the Xavantina-Cachimbo Expedition area. Phil. Trans. R. Soc. B 266: 449-492.

Ratter, J. A., Askew, G. P., Montgomery, R. F. \& Gifford, D. R. (1977). Observações adicionais sobre o cerradão de solos mesotróficos no Brasil Central. In: FerR I, M. G. (co-ordinator) IV Simpósio Sobre o Cerrado, pp. 306-316. São Paulo: EDUSP.

Ratter, J. A., Askew, G. P., Montgomery, R. F. \& Gifford, D. R. (1978). Observations on the vegetation of northeastern Mato Grosso. II. Forests and soils of the Rio Suiá-Missú area. Proc. R. Soc. Lond. B 203: 191-208.

Silva-Júnior, M. C., Felfili, J. M., Nogueira, P. E. \& Rezende, A. V. (1998). Análise florística das matas de galeria no Distrito Federal. In: Ribeiro, J. F. (ed.) Cerrado: matas de galeria, pp. 53-84. Brasília: EMBRAPA-CPAC.

W Alter, B. M. T. (1995). Distribuição espacial de espécies perenes em uma mata de galeria inundável no Distrito Federal; florística e fitossociologia. MSc thesis, Universidade de Brasília, Brasília.

ZAR, J. H. (1999). Biostatistical Analysis, 4th edition. New Jersey: Prentice-Hall. 